\title{
EDITORIAL
}

\section{¿Qué publicación del 2020 no dejaría de leer?}

El tratamiento quirúrgico del cáncer de recto atraviesa un momento de debate, debido a que en la actualidad disponemos de cuatro tipos de abordaje diferentes: abierto, laparoscópico, robótico y transanal. Este último, representado por el acrónimo TaTME, se encuentra en una etapa de revisión y análisis. Es por ello que elegí este artículo publicado en el mes de mayo en Colorectal Disease.

Consensus Statement

\section{International expert consensus guidance on indications, implementation and quality measures for transanal total mesorectal excision}

\begin{abstract}
The TaTME Guidance Group representing the ESCP (European Society of Coloproctology), in collaboration with the ASCRS (American Society of Colon and Rectal Surgeons), ACPGBI (Association of Coloproctology of Great Britain and Ireland), ECCO (European Crohn's and Colitis Organisation), EAES (European Association of Endoscopic Surgeons), ESSO (European Society of Surgical Oncology), CSCRS (Canadian Society of Colorectal Surgery), CNSCRS (Chinese Society of Colorectal Surgery), CSLES (Chinese Society of Laparo-Endoscopic Surgery), CSSANZ (Colorectal Surgical Society of Australia and New Zealand), JSES (Japanese Society of Endoscopic Surgery), SACP (Argentinian Society of Coloproctology), SAGES (Society of American Gastrointestinal and Endoscopic Surgeons), SBCP (Brazilian Society of Coloproctology), SwissMIS (Swiss Association for Minimally Invasive Surgery) $\mathbf{x}$
\end{abstract}

Colorectal Dis. 2020 Jul; 22(7): 749-755.

doi: 10.1111/codi.15147. Epub 2020 Jul 13. PMID: 32441803.

La elección de este artículo se basa en tres aspectos principales. En primer lugar este consenso refleja la activa colaboración de un grupo de cirujanos colorrectales con especial interés en el desarrollo e implementación de esta nueva vía de abordaje. En segundo lugar, este grupo representa a su vez a 15 prestigiosas entidades internacionales entre las que se encuentra la Sociedad Argentina de Coloproctogía (SACP). Y en tercer lugar, y probablemente el punto más importante, porque su objetivo es el de ofrecer una guía para la implementación segura de este técnica quirúrgica.

Empleando el método Delphi y reuniones presenciales, se identificaron primeramente 4 aspectos fundamentales relacionados a la implementación segura de la TaTME:

1. sus indicaciones,

2. las medidas de calidad del procedimiento y sus resultados,

3. el entrenamiento previo requerido y

4. sus aspectos técnicos.

Entre los enunciados destacados que lograron mayor nivel de consenso, surge que la TaTME podría ser empleada en patología benigna como maligna en aquellos casos que anticipadamente se prevé cierta dificultad técnica durante la disección pelviana. A su vez destaca que cada institución debería establecer un protocolo de armado y disposición del quirófano para estos procedimientos, como así también del manejo perioperatorio de estos pacientes. Otra recomendación con fuerte nivel consenso hace referencia a que aquellos cirujanos que quieran comenzar a implementar esta técnica deberían completar un proceso de entrenamiento estructurado y que a su vez sus primeros casos sean supervisados por un "proctor". Y, por último, establece con un elevado nivel de acuerdo que el equipo quirúrgico debería estar conformado por dos cirujanos colorrectales, donde al menos uno de ellos tenga experiencia en TaTME y abordaje miniinvasivo del cáncer de recto. 
REV ARGENT COLOPROCT | 2020 | VOL. 32, № 1

DOI: $10.46768 /$ racp.v32i01.119

Si bien estos consensos carecen de un adecuado nivel de evidencia, en este caso resalta cuál debería ser el ámbito adecuado para implementar de manera correcta esta técnica quirúrgica. Es por ello que recomiendo su lectura.

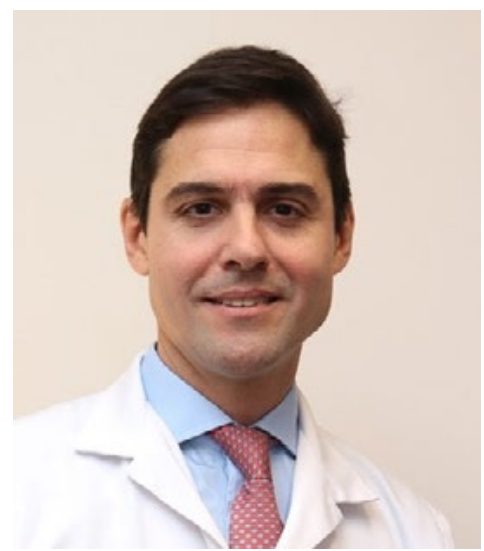

Gustavo Rossi

Hospital Italiano de Buenos Aires. Ciudad Autónoma de Buenos Aires, Argentina 